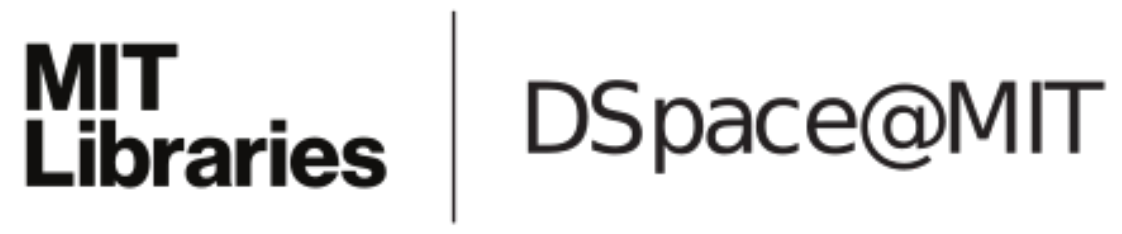

\author{
MIT Open Access Articles
}

\section{Impact of a Centrebody On the Unsteady Flow Dynamics of a Swirl Nozzle: Intermittency of PVC Oscillations}

The MIT Faculty has made this article openly available. Please share how this access benefits you. Your story matters.

Citation: Gupta, Saarthak, Shanbhogue, Santosh, Shimura, Masayasu, Ghoniem, Ahmed F and Hemchandra, Santosh. 2021. "Impact of a Centrebody On the Unsteady Flow Dynamics of a Swirl Nozzle: Intermittency of PVC Oscillations." Journal of Engineering for Gas Turbines and Power, 144 (2).

As Published: 10.1115/1.4052144

Publisher: ASME International

Persistent URL: https://hdl.handle.net/1721.1/138758

Version: Final published version: final published article, as it appeared in a journal, conference proceedings, or other formally published context

Terms of Use: Article is made available in accordance with the publisher's policy and may be subject to US copyright law. Please refer to the publisher's site for terms of use. 


\author{
Saarthak Gupta ${ }^{1}$ \\ Department of Aerospace Engineering, \\ Indian Institute of Science, \\ Bangalore 560012, India \\ e-mail: gsaarthak@iisc.ac.in
}

\section{Santosh Shanbhogue}

Department of Mechanical Engineering, Massachusetts Institute of Technology,

Cambridge, MA 02139 e-mail: santosh1@mit.edu

\author{
Masayasu Shimura \\ Department of Mechanical Engineering. \\ Tokyo Institute of Technology, \\ Meguro-ku, \\ Tokyo 1528550, Japan \\ e-mail: shimura.m.aa@m.titech.ac.jp
}

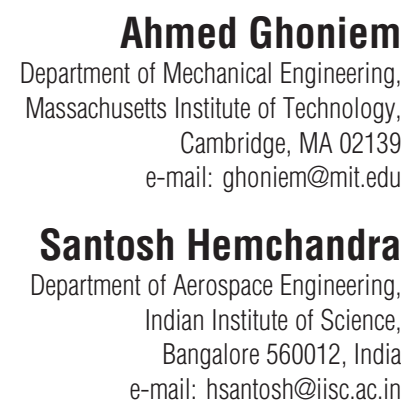

\section{Impact of a Centerbody on the Unsteady Flow Dynamics of a Swirl Nozzle: Intermittency of Precessing Vortex Core Oscillations}

The precessing vortex core $(P V C)$ is a self-excited flow oscillation state occurring in swirl nozzles. This is caused by the presence of a marginally unstable hydrodynamic helical mode that induces precession of the vortex breakdown bubble (VBB) around the flow axis. The PVC can impact emissions and thermoacoustic stability characteristics of combustors in various ways, as several prior studies have shown. In this paper, we examine the impact of centerbody diameter $\left(D_{c}\right)$ on the PVC in a nonreacting flow in a single nozzle swirl combustor. Time-resolved high-speed stereoscopic PIV measurements are performed for combinations of two swirl numbers, $S=0.67$ and 1.17 and $D_{c}=9.5 \mathrm{~mm}$, $4.73 \mathrm{~mm}$, and 0 (i.e., no centerbody). The bulk flow velocity at the nozzle exit plane is kept constant as $U_{b}=8 \mathrm{~m} / \mathrm{s}$ for all cases (Re $\left.\sim 20,000\right)$. The centerbody end face lies in the nozzle exit plane. A new modal decomposition technique based on wavelet filtering and proper orthogonal decomposition provides insight into flow dynamics in terms of global modes extracted from the data. The results show that without a centerbody, a coherent PVC is present in the flow as expected. The introduction of a centerbody makes the PVC oscillations intermittent. These results suggest two routes to intermittency as follows. For $S=0.67$, the VBB and centerbody wake recirculation zone regions are nominally distinct. Intermittent separation and merger due to turbulence result in PVC oscillations due to the destabilization of the hydrodynamic VBB precession mode of the flow. In the $S=1.17$ case, the time averaged VBB position causes it to engulf the centerbody. In this case, the emergence of intermittent PVC oscillations is a result of the response of the flow to broadband stochastic forcing imposed on the time averaged vorticity field due to turbulence. [DOI: 10.1115/1.4052144]

\section{Introduction}

Swirl flows are used in gas turbine combustors to achieve good fuel-air mixing and reliable flame stabilization [1]. Air enters the combustor primary zone through one or more nozzles equipped with passages or vanes that impart a swirling motion to the flow. The characteristics of these types of flows are determined by the swirl number, $S$, defined as the ratio of the streamwise fluxes of azimuthal and axial flow momentum. For sufficiently high values of $S$, breakdown of the axial vortex can result in flow structures of many different forms depending on the value of $S$ and boundary conditions [2-5]. For the range $0.5 \leq S \leq 1$, vortex breakdown typically leads to the formation of a nominally axisymmetric bubble-like recirculation zone in the flow, referred to commonly as the vortex breakdown bubble (VBB). The presence of the VBB creates a stagnation point in the flow at its upstream end. Additionally, flow recirculation brings hot products and reaction intermediates in contact with the fresh unburnt gas. Both of these reasons aid flame stabilization downstream of the nozzle.

The VBB deflects the oncoming flow around itself to form an annular jet. Strong shear layers are formed between the annular jet and the VBB on the side closer to the flow axis, as well as, between the annular jet and the surrounding fluid on the other side. When created this way, several past studies have shown that the emergence of the VBB can be accompanied by self-excited precession, i.e., the VBB's axis rotates around the geometric axis of the flow [6-8]. Manoharan et al. [8] showed from their weakly

\footnotetext{
${ }^{1}$ Corresponding author.

Manuscript received July 20, 2021; final manuscript received August 2, 2021; published online November 8, 2021. Editor: Jerzy T. Sawicki.
}

nonlinear analysis of a variable swirl turbulent jet experiment that this is due to the emergence of a marginally unstable linear helical hydrodynamic mode of the flow, due to the onset of vortex breakdown. The amplitude distribution of this mode induces VBB precession and resulting stable limit cycle oscillation, and perturbs the flow shear layers in a helical fashion. Their rollup results in the creation of a helical vortex that is wrapped around the precessing VBB. This self-excited flow structure is commonly referred to as the precessing vortex core (PVC) [7-10].

The PVC is known to influence combustor operation through its influence on unsteady flame behavior in several ways. For instance, the presence of a PVC can provide low-velocity pathways for flame propagation that can result in macroscopic flame shape changes in the combustor [11-14]. This can, in turn, alter the spatial distribution of heat release within the combustor and result in the onset of undesirable thermoacoustic oscillations $[11,14]$. These acoustic pressure oscillations are driven by unsteady heat release oscillations driven by the response of the flame to velocity and fuel-air ratio oscillations that the acoustic pressure oscillations induce. Thus, by altering the flame shape and, thereby, the spatial distribution of heat release, the PVC can cause the emergence of thermoacoustic oscillations $[11,12,14]$. On the other hand, controlling PVC dynamics by adding microjets that introduce counter-rotating swirl has been successful in suppressing thermoacoustic instability by changing the flow and overall flame macrostructure $[15,16]$.

In some cases, the PVC is known to have the opposite effect and suppress thermoacoustic oscillations. An ongoing study extending the weakly nonlinear analysis of Manoharan et al. [8] to include imposed forcing suggests that this is due to nonlinear coupling between the PVC and the axisymmetric hydrodynamic 
response of the flow to the forcing imposed by thermoacoustics. These results will be presented in future papers. PVCs are also known to have a favorable impact on combustor emissions $[17,18]$. This is attributed to the enhancement of fuel-air mixing that the PVC generates. For all these reasons, it is clear that from a technological standpoint, the emergence and dynamics of PVC oscillations in practical combustors need to be managed. Therefore, understanding PVC behavior and how its emergence may be controlled in combustors are problems of significant technological importance.

Gas turbine combustor nozzles are equipped with centerbodies for various reasons such as providing a means for fuel injection and improving flame stabilization. Several recent studies have shown that the presence of centerbodies can result in the stabilization of PVC oscillations and, thereby, flame stabilization $[19,20]$. The nonlinear and linear analyses of Manoharan et al. [8] and Tammisola et al. [21], respectively, show that limit cycle amplitude growth and linear instability of the VBB precession mode are driven by flow processes in the region upstream of the VBB, referred to as the "wavemaker." The presence of the wavemaker has subsequently been confirmed in several other studies as well $[13,19,20]$. The recent studies of Kaiser et al. [20] and Mukherjee et al. [19], using a combination of experimental measurements, large eddy simulations (LES), and linear stability analysis, show that the introduction of a centerbody upstream of the VBB disrupts the wavemaker and potentially stabilizing the VBB precession mode and suppressing the PVC oscillations. This is consistent with observations reported in early variable swirl jet studies of Escudier and Keller [6], who report the emergence of a precessing VBB with increasing $S$. They report qualitatively that the movement of the VBB upstream with increasing $S$ suppresses $\mathrm{VBB}$ precession when it reaches the upstream centerbody.

Figure 1 shows the two types of time averaged flow states that are possible when a centerbody is introduced into a postvortex breakdown swirl flow. These two states differ in the time averaged position of the VBB relative to the centerbody. In the first case, the VBB position is downstream of the centerbody end-face resulting in the formation of a centerbody wake recirculation zone (CWRZ), as shown schematically in Fig. 1(a). We refer to this as the disjoint case in this paper to emphasize that the VBB and the CWRZ are nominally separate flow regions. Mukherjee et al. [19] showed from a combination of LES and linear stability analysis that when this state is created in the flow, the linear VBB precession mode is destabilized, and limit cycle PVC oscillations emerge. The second case is when the VBB position is sufficiently upstream, so as to merge with the CWRZ or even engulf the centerbody, as shown schematically in Fig. 1(b). We refer to this time

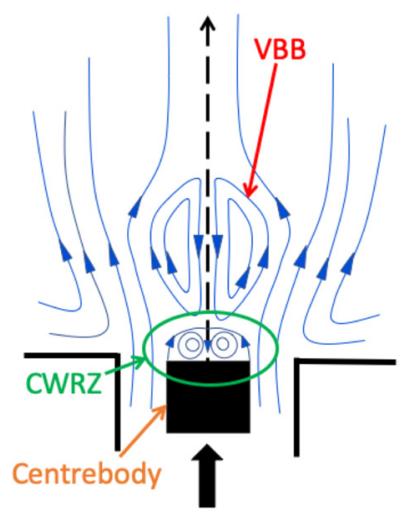

(a)

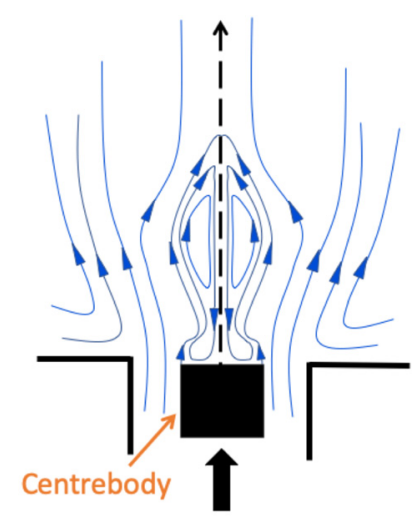

(b)
Fig. 1 Schematic of the two possible time averaged flow states that can be achieved in swirling jets based on the spatial position of the VBB and CWRZ relative to each other: (a) disjoint (separated) and (b) conjoined (merged) the averaged flow state as the conjoined case. In this case, the merger of the VBB with the CWRZ or even the physical presence of the centerbody when engulfed by the VBB disrupts the wavemaker of the VBB precession mode [19,20]. This then suggests that the VBB precession should be suppressed. However, it is unclear as to how effective the suppression of VBB precession by a centerbody is in practice, even though Kaiser et al. [20] broadly show suppression of the VBB precession in their experiment.

Therefore, in this paper, we study the eventual flow state that results in the above two cases and assess the effectiveness with which the suppression of VBB precession, predicted theoretically, occurs in practice. To this end, we study the unsteady dynamics of the flow that results from both of these conditions in the single nozzle swirl experiment of Ghoniem and coworkers [11,12]. The time averaged position of the VBB is varied relative to the nozzle exit plane by changing the upstream swirl number. This is achieved by using two fixed vane axial swirlers with different vane angle settings. The swirlers are equipped with a cylindrical centerbody whose length ensures that its end-face is flush with the combustor dump plane. Time resolved stereoscopic particle image velocimetry (sPIV) measurements of the flow field are performed for three cases for each upstream swirl setting. Two of these are for centerbodies with diameter, $D_{c}=9.5 \mathrm{~mm}$ and $4.17 \mathrm{~mm}$, and the third is by leaving out the centerbody entirely, i.e., $D_{c}=0.0 \mathrm{~mm}$. Note that changing $D_{c}$ changes the nominal axial length of the CWRZ in the disjoint case and, thereby, influences its ability to merge with the VBB downstream.

The flow cases with centerbodies in the present experiments show intermittent PVC oscillations, i.e., they are not completely suppressed as prior studies suggest $[19,20]$. A newly developed modal decomposition technique, using a combination of continuous wavelet transforms and proper orthogonal decomposition (POD) [22-24] - referred to here as wavelet POD (WPOD), is used to gain insight into the globally nonstationary, intermittent dynamics of the flow. We show that the presence of a centerbody can induce intermittent PVC oscillations through two routes. In the disjoint case, intermittent separation and merger between the CWRZ and VBB result in intermittent VBB precession and PVC oscillations. In the conjoined case, stochastic forcing by background turbulence results in an intermittent hydrodynamic response due to the VBB mode. Thus, even though the linear hydrodynamic VBB mode is nominally stabilized, PVC oscillations can occur due to the presence of background turbulence.

The rest of this paper is organized as follows. Sec. 2 describes the experimental setup and details the cases studied in this paper. Sec. 3 describes the data analysis procedures including the new WPOD procedure. Sec. 4 presents the results of this analysis, and Sec. 5 concludes the paper with an outlook on future work.

\section{Experimental Setup}

Figure 2 illustrates the single nozzle swirl combustor experimental setup used in this study. Air enters the setup through a circular inlet duct with an inner diameter of $D=38 \mathrm{~mm}$ through a choke plate, as shown on the left in Fig. 2. The inlet duct then undergoes a sudden expansion to the combustor section, whose inner radius is nearly twice that of the inlet duct. The origin of coordinates in this study is positioned on the centerline of the

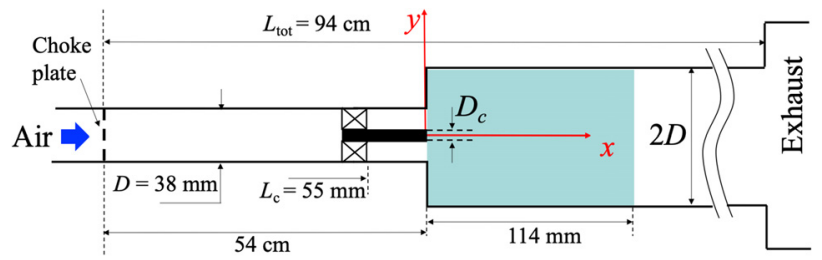

Fig. 2 Schematic of the single nozzle combustor facility showing details of the centerbody and swirler 
geometry at the dump plane. The $x$-axis is chosen to lie along the streamwise flow axis. An axial swirler is placed in the inlet duct, such that its central hub is $5.5 \mathrm{~cm}$ upstream of the origin. The swirler is comprised of eight filleted plates set at an angle to the oncoming flow in order to induce swirl. A cylindrical centerbody whose end face is flush with the dump plane is attached to the central hub of the swirler, as shown schematically in Fig. 2. The mass flowrate of air into the setup is controlled using a Sierra instruments 780S Flat-Trak flowmeter with an accuracy of $\pm 1 \%$ of maximum capacity.

The combustor section wall is a cylindrical fused silica quartz tube to allow for optical access. Flow measurements are made using two-dimensional, three-component high-speed sPIV. These measurements are obtained in a field of view that encompasses the entire width of the combustor, starting from the dump plane up to a downstream distance of $114 \mathrm{~mm}(3 D)$, as shown schematically by the green region in Fig. 2. The illumination for these measurements is provided by a Photonics dual-head $50 \mathrm{~W}$ Nd:YLF laser, using standard diverging sheet generation optics. The flow region is imaged with a pair of $1024 \times 1024$ pixel, high-speed Nova S6 CMOS (Photron, CA) cameras using F-mount Nikon $60 \mathrm{~mm}$ lenses equipped with schiempflug adapters.

The cameras are placed on both sides of the light sheet, as shown in Fig. 3. One camera collects particle images in the forward scatter mode with a larger intensity, and another in the backward scatter mode with lower intensity. The aperture on both cameras is, thus, set to $f=8.0$ and 5.6, respectively, to account for this difference. Both cameras are placed roughly $500 \mathrm{~mm}$ away from the imaging plane and inclined approximately $45 \mathrm{deg}$ to the imaging plane normal. The scheimpflug adapters were adjusted to bring the entire region of interest into focus. A three dimensional image with a precise arrangement of $5 \mathrm{~mm}$ diameter dots was used to calibrate the stereo system. A total of 2048 pairs of images were acquired at $2 \mathrm{kHz}$ to yield $1.024 \mathrm{~s}$ worth of time resolved snapshots.

The particle images are processed using Lavision's DAvIS software (version 10) using the following series of operations: First, all four images (two image pairs) are background subtracted using images with no flow, but with laser illumination. Next, all images are subject to a sliding background filter eight pixels wide. Velocities are computed from these image pairs in five iterative stepsinitially with a $64 \times 64$ window with a $50 \%$ overlap, then using four passes with a $32 \times 32$ window also with a $50 \%$ overlap. Finally, the data are median filtered with a $1 \times, 5 \times 5$ window. If the resulting velocity vector has a correlation coefficient less than 0.5 , it is considered untrustworthy and deleted. These operations result in velocity field data with a spatial resolution of $1.8 \mathrm{~mm}$ in both $x$ and $y$ directions.

2.1 Operating Conditions. Flow field measurements were made for two swirler blade angles $\alpha_{b}=45 \mathrm{deg}$ and $60 \mathrm{deg}$ and three values of centerbody diameter $D_{c}=9.5 \mathrm{~mm}, 4.73 \mathrm{~mm}$, and $0 \mathrm{~mm}$ (no centerbody), for each blade angle. Thus, the swirl

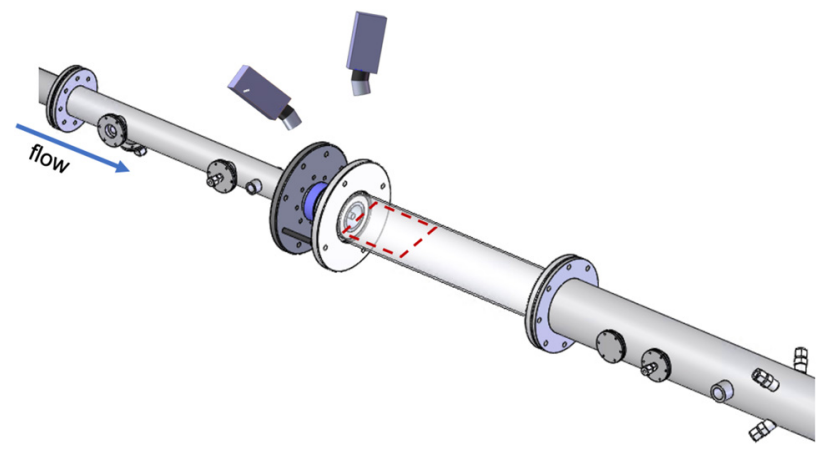

Fig. 3 Illustration of the arrangement of cameras in the stereoconfiguration and the imaging region (red line) number $S$ associated with $\alpha_{b}=45 \mathrm{deg}$ and $60 \mathrm{deg}$, respectively, is 0.67 and 1.17 , determined using the following formula [25]

$$
S=\frac{2}{3}\left(\frac{1-\left(D_{h} / D\right)^{3}}{1-\left(D_{h} / D\right)^{2}}\right) \tan \alpha_{b}
$$

where $D_{h}$ is the diameter of the hub. All measurements were obtained for a fixed area averaged bulk flow velocity, $U_{b}=8 \mathrm{~m} / \mathrm{s}$. This was achieved using different upstream air flowrate settings for the effective annular area associated with each value of $D_{c}$ at the exit of the inlet duct. Thus, the flow Reynolds number, $\operatorname{Re}=U_{b} D / \nu \sim 20,000$. The various combinations of swirl number $S$ and centerbody geometries studied in this work are shown in Table 1.

The choice of these cases was motivated by the following considerations. Cases 1 and 4 in Table 1 are used to establish baseline nozzle behavior without a centerbody. The change in the value of $S$ between cases 1 and 4 yields two different time averaged positions of the vortex breakdown bubble (VBB) with respect to the dump plane. It is well known that increasing $S$ causes the VBB to move upstream (see for e.g., Ref. [6]). As will be shown later, this nominally results in the $S=0.67$ case being a disjoint case and $S=1.17$ being a conjoined case. $D_{c}$ is varied for both cases in order to understand its impact on the eventual flow state.

\section{Data Analysis}

We analyze the sPIV data obtained from the various cases in Table 1 in broadly three different ways. The first is by examining traditional time averaged means and variances of flow velocity components in order to understand the effect of changing $S$ and $D_{c}$ on the overall spatial structure of the flow field. The second is by performing spectral proper orthogonal decomposition (SPOD) of the dataset to verify the presence of coherent oscillations. The third is using a new multiresolution method based on combining wavelet decompositions of the data with POD, which we refer to as WPOD in this paper. We present a brief discussion of POD, SPOD, and WPOD methods in general, in order to highlight the sort of flow dynamics that each approach may be expected to reliably capture. We begin with a brief description of POD that forms the basis of SPOD and WPOD.

3.1 Proper Orthogonal Decomposition. Proper orthogonal decomposition is a data decomposition technique that is used to determine a set of mutually orthogonal basis functions and associated components that reconstruct the data [26-29]. When applied to time resolved flow field measurements like in the present paper, the basis modes identify the most energetic unsteady flow field structures within the dataset. Thus, assuming that twodimensional snapshots of flow field measurements are available at discrete points in space, e.g., from sPIV measurements, POD determines a decomposition of the data that take the following form:

$$
q(x, y, t)=\sum_{k=0}^{N} a_{k}(t) \phi_{k}(x, y)
$$

Table 1 Centerbody geometry parameters investigated in this study

\begin{tabular}{lccc}
\hline \hline Case & $S$ & $L_{c}$ & $D_{c}$ \\
\hline 1 & 0.67 & $55 \mathrm{~mm}$ & $0 \mathrm{~mm}$ \\
2 & 0.67 & $55 \mathrm{~mm}$ & $4.7 \mathrm{~mm}$ \\
3 & 0.67 & $55 \mathrm{~mm}$ & $9.5 \mathrm{~mm}$ \\
4 & 1.17 & $55 \mathrm{~mm}$ & $0 \mathrm{~mm}$ \\
5 & 1.17 & $55 \mathrm{~mm}$ & $4.7 \mathrm{~mm}$ \\
6 & 1.17 & $55 \mathrm{~mm}$ & $9.5 \mathrm{~mm}$ \\
\hline \hline
\end{tabular}


where $q(x, y, t)$ is the any flow quantity, e.g., velocity components in the case of SPIV measurements, $\phi_{k}$ is the POD modes, and $a_{k}$ is the temporal variations associated with each of these modes. By construction, POD ensures that the modes $\phi_{k}$ are mutually orthogonal, i.e.,

$$
\left\langle\phi_{i}, \phi_{j}\right\rangle=\int_{\Omega} \phi_{i} \phi_{j} d \Omega=\delta_{i j}
$$

where $\delta_{i j}$ is the Kronecker delta, and the integration, appropriately discretized, spans the spatial region over which measurements are available.

The POD basis modes $\phi_{k}$ are also optimal, i.e., they minimize the error: $\left\|q-q_{M}\right\|=\left(\left\langle\left(q-q_{M}\right),\left(q-q_{M}\right\rangle\right)^{1 / 2}\right.$, where $q_{M}$ is an approximate reconstruction of $q$ that retains contributions from only the first $M$ modes, i.e., first $M$ terms in Eq. (2).

The POD modes $\phi_{k}$ are determined directly from the data $q(x, y, t)$ as follows. A snapshot matrix $\mathbf{S}$ is created from successive flow field snapshots $q(x, y, t)$, where each column is a snapshot of $q$ over the measurement domain at a given time $t$, re-arranged as a column vector. The snapshots are assumed to be equispaced in time in this paper. Assuming that there are $P$ measurements per snapshot and $Q$ snapshots in all; $\mathbf{S}$ is a matrix of size $P \times Q$. It is now possible to determine the singular value decomposition (SVD) of $\mathbf{S}$ as follows:

$$
\mathbf{S}=\boldsymbol{\Phi} \boldsymbol{\Sigma} \mathbf{A}^{T}
$$

where $\boldsymbol{\Phi}$ is a $P \times P$ matrix whose columns are the modes $\phi_{k}, \boldsymbol{A}$ is a $Q \times Q$ matrix whose columns sample $a_{k}(t)$ in Eq. (2) at the times corresponding to each snapshot, and $\boldsymbol{\Sigma}$ is $P \times Q$ matrix whose upper square $P \times P$ submatrix is a diagonal matrix of singular values, i.e., $\boldsymbol{\Sigma}^{P \times P}=\operatorname{diag}\left[\sigma_{1}, \ldots, \sigma_{P}\right]$. The value of $\sigma_{k}^{2}$ quantifies the contribution of the mode $\phi_{k}(x, y)$ to the total energy in $q(x, y, t)$. The above process orders the modes $\phi_{k}$ in descending order of the values of $\sigma_{k}^{2}$.

Since the POD is applied on snapshots of time series data alone, the temporal variations, $a_{k}(t)$, can, in general, be composed of multiple frequency components, i.e., it is not possible to associate the $\phi_{k}$ with flow motions at a given frequency. In many cases, such as when analyzing flows with coherent flow motions like a PVC with a well-defined frequency or when a spectrally resolved basis for the reconstruction of flow motions is needed, a method that can retain the optimal basis property of the POD modes while being spectrally selective is desirable. We now describe two modifications to the above POD procedure that allow for spectrally resolved optimal basis modes to be determined for both stationary and nonstationary data.

3.2 Spectral Proper Orthogonal Decomposition. The SPOD, proposed by Towne et al. [30], extracts optimal energy ordered orthogonal modes that are spectrally resolved. This is accomplished by performing a Fourier decomposition of the data first and then applying the POD to snapshots at each frequency. Complete details of this method can be found in Towne et al. [30], and we limit our description here to a broad overview of the method.

The time series data are first divided into $M$ ensembles of $L$ consecutive snapshots. A discrete Fourier transform (DFT) is applied to the time series at each spatial point to obtain the field $\hat{q}^{m, L}(x, y ; \omega)$, where $m$ represents the ensemble index. This process yields flow snapshots at $L / 2$ discrete values of frequency, $\omega$ from each ensemble. A snapshot matrix at a given frequency, $\omega$, resolved by the DFT is constructed using $\hat{q}^{m, L}(x, y ; \omega)$ from each ensemble, mapped into column vectors as the columns of a matrix $\hat{\mathbf{S}}(\omega)$ of size, $P \times M$. The SVD in Eq. (3) is then computed for $\hat{\mathbf{S}}\left(\omega_{l}\right)$ to yield POD modes $\hat{\phi}_{k}\left(x, y ; \omega_{l}\right)$ and their corresponding singular values $\hat{\sigma}_{k, \omega_{l}}$.

Since $\hat{\phi}_{k}(x, y)$ are now computed using Eq. (4) on $\hat{\mathbf{S}}(\omega)$ comprised of Fourier components at $\omega$ alone, they are readily interpreted as POD modes that reconstruct flow dynamics at $\omega$, i.e., $\hat{q}(x, y, t ; \omega)$, as follows:

$$
\hat{q}(x, y, t ; \omega)=\sum_{k=0}^{M} \hat{a}_{k} \hat{\phi}_{k}(x, y ; \omega) e^{-i \omega t}+c . c .
$$

where $i=\sqrt{-1}$ and c.c. are the complex conjugate of the first summation on the right in Eq. (5). The above approach is suited for underlying flow data comprised of stationary unsteady flows, i.e., whose spectral content does not change with time. This is the underlying assumption made by using the Fourier transform on the measured unsteady data.

We briefly review here an alternative approach to achieving spectral selectivity with the POD method formulated by Sieber et al. [31]. This method applies filtering to the diagonals of the correlation matrix, $\mathbf{K}=\mathbf{S}^{\mathrm{T}} \mathbf{S}$. The idea here is to reduce the frequency content of $\mathbf{K}$ before computing its eigenvectors to yield basis modes. This is motivated by the fact that the more close $\mathbf{K}$ is to a Toeplitz circulant form, the more close are the POD modes to a discrete Fourier transform of the time series data [32]. However, the POD modes obtained are not orthogonal as depending on the filter width chosen, one obtains modes that approximate the optimal POD modes or modes resulting from a DFT of the underlying data. This is not the case in the method of Towne et al. [30] described here because the raw data itself are decomposed into Fourier modes, and the POD is applied on these, thereby, ensuring an optimal and spectrally selective decomposition.

However, many flows are characterized by intermittent unsteady events that can be localized in both frequency and time. Fourier decomposition replaces the description of signal variation in time with a description of signal variation in frequency space. Therefore, the Fourier transform cannot describe a signal whose spectral content varies with time. Therefore, for this type of flow data, a frequency-time decomposition that wavelet transforms provide is an appropriate approach.

3.3 Wavelet Proper Orthogonal Decomposition. This approach replaces the initial spectral decomposition in the SPOD approach with a frequency-time decomposition of $q(x, y, t)$ using wavelet transforms [33]. This frequency-time decomposition effectively captures flow features, such as intermittent flow oscillation events that are of limited extent in both frequency and time. Time series signals corresponding to specific frequency ranges, in which intermittent oscillations of interest occur can now be reconstructed from the wavelet decomposed time series data. This is achieved by setting the wavelet coefficients outside the frequency range of interest to zero and inverting the transform to yield a time varying signal containing spectral content from the frequency band of interest alone. We refer to this process as wavelet filtering.

Wavelet filtering can now be combined with POD to extract basis modes and temporal variations that characterize intermittent flow behavior in $q(x, y, t)$ as follows. First, the frequency band of interest for wavelet filtering is identified by examining the wavelet transforms of $q(x, y, t)$ at one or more points of interest in the flow field. Next, wavelet filtering is applied at all points in the flow field to reconstruct the time variation of the flow field as whole, within this frequency band. This yields wavelet filtered snapshots, $\tilde{q}(x, y, t)$, of the original $q(x, y, t)$ measurement that isolate the intermittent flow oscillations of interest. Next, POD modes are determined from Eq. (4), using a snapshot matrix $\tilde{\mathbf{S}}$ constructed using $\tilde{q}(x, y, t)$. Thus, $\tilde{q}(x, y, t)$ can be written in terms of its POD modes, $\tilde{\phi}_{k}(x, y)$, as follows:

$$
\tilde{q}(x, y, t)=\sum_{k=0}^{N} a_{k}(t) \tilde{\phi}_{k}(x, y)
$$

We refer to the POD modes, $\tilde{\phi}_{k}$, as WPOD modes in this paper. The wavelet filtering step ensures that these modes capture 
information only contained within the frequency band used for filtering. Furthermore, since POD does not require $a_{k}(t)$ to have the form of a stationary harmonic oscillation, the decomposition in Eq. (6) can recover the spatial distribution of flow oscillations associated with intermittent flow oscillation events.

Several approaches employing this technique have been described in recent studies based on discrete wavelet transforms [24], discrete wavelet packet transforms [23], and discretizations of the continuous wavelet transform (CWT) [22]. We adopt the latter approach in this paper for the following reasons. The CWT discretizes the scale parameter (and hence frequency) in the wavelet basis as $2^{j / v} j=0,1,2, \ldots$, with the choice $\nu=10$ in this paper. This approach provides a frequency-time decomposition of $q(x, y, t)$ with a finer frequency resolution than discrete wavelet transforms, while retaining the time resolution of the original data. Furthermore, the CWT is a shift invariant transform, i.e., shifts in the original signal translate into shifts of the same extent in the CWT. This ensures that phase relationships between various frequency components in the original signal are preserved in the computed transform. These properties of the CWT make it suitable for accurate resolution of intermittent flow dynamics that are compact in frequency space. However, the tradeoff in this approach is the higher computational cost of computing a CWT.

We use the continuous forward and inverse wavelet transform implementations provided by the "cwt" and "icwt" MATLAB functions in this paper for wavelet filtering. We choose to use the bump wavelet because it allows for better frequency resolution in the computed transform. Using other analytic wavelets like Morlet, etc. did not result in significantly different results for the data analyzed in this work.

3.4 Wavelet Proper Orthogonal Decomposition: Example Analysis. We now show results from each of the three types of modal decompositions applied to the sPIV dataset from case 4 (see Table 1). Figure 4 shows the scalogram of the transverse velocity component $\left(u_{y}\right)$, determined using the CWT at $x / D=$ 0.28 on the geometric centerline for this case. Time and frequency have been normalized using $D$ and $U_{b}$. The field in Fig. 4 shows the magnitude of the wavelet coefficients. It is clear from Fig. 4 that there is an intermittent oscillation at $\mathrm{St}=f D / U_{b} \sim 0.8$. We now apply wavelet filtering to retain a band of width $\Delta \mathrm{St}=0.4$ around this peak value at every point in the domain and compute the POD of the resulting wavelet filtered snapshots.

Figure $5(a)$ shows the modal energy spectrum, i.e., the variation of modal energy, $\tilde{\sigma}_{k}^{2}$, across the computed WPOD modes (black circles). Also shown for comparison are the modal spectra obtained from the application of POD (red squares) directly (i.e., without any filtering) and SPOD (blue crosses) at $\mathrm{St}=0.8$, of the same data. The SPOD results have been obtained using $M=9$ ensembles of $L=400$ snapshots, with a $50 \%$ overlap between successive ensembles. The SPOD procedure yields complex valued

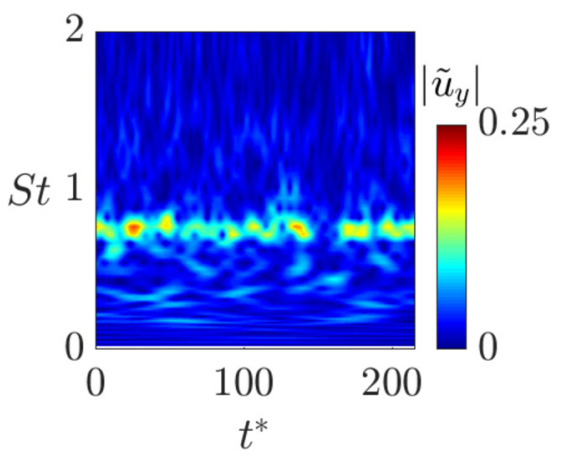

Fig. 4 Wavelet scalogram of the transverse velocity $\left(u_{y}\right)$ on the geometric centerline at $x / D=0.28$ for case $4 \quad(S=1.17$, $D_{c}=9.5 \mathrm{~mm}$ )

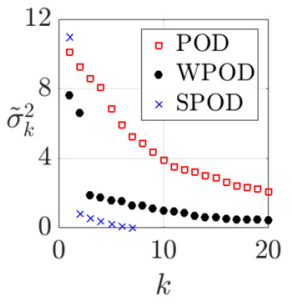

(a)

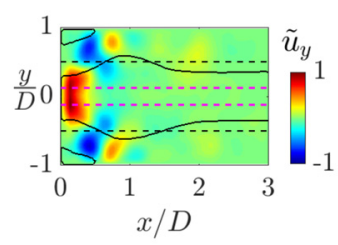

(c)

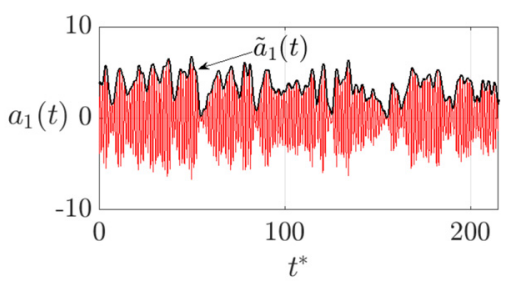

(b)
Fig. 5 WPOD decomposition around $S t=0.8$ for case 4 $\left(S=1.17, D_{c}=9.5 \mathrm{~mm}\right.$ ): (a) WPOD modal spectrum (black circles). (b) Temporal variation of mode 1. (c) Mode shape $\tilde{\phi}_{1}$. (d) Instantaneous amplitude phase space plot (blue crosses) and the red contour show the region in instantaneous amplitude phase space visited by the trajectory $90 \%$ of the time within the measured time record. Also shown in (a) is the modal energy spectrum from POD applied to the unfiltered raw data (squares).

modes, whose real and imaginary parts are equivalent to the real POD and WPOD modes. Note that both SPOD and WPOD results show that most of the energy of the oscillations is captured by the first mode and the first two modes, respectively. The POD result, on the other hand, shows a nearly continuous variation of modal energy across modes and does not isolate the flow oscillations of interest in the same way that WPOD and SPOD do. The use of an initial Fourier decomposition in SPOD, however, leads to the interpretation of the result from SPOD in Fig. 5(a) that this mode represents a stationary global oscillation of the flow at $\mathrm{St}=0.8$. However, Figs. 5(b) and 5(c) shows the temporal variation of $a_{1}(t)$ and associated mode shape $\tilde{\phi}_{1}(x, y)$, respectively, of the most energetic WPOD mode. The instantaneous amplitude of $a_{1}(t)$ is defined as the magnitude of the Hilbert transform of $a_{1}(t)$ as follows:

$$
\tilde{a}_{1}(t)=\left|\frac{1}{\pi} \int_{-\infty}^{\infty} \frac{a_{1}(\tau)}{t-\tau} d \tau\right|
$$

The thick black curve in Fig. 5(b) shows the variation of $\tilde{a}_{1}(t)$, which, as may be expected, is simply the amplitude envelope of $a_{1}(t)$. We use the "Hilbert" function provided by MATLAB to compute Hilbert transforms in this paper.

Note that Fig. 5(b) shows that $a_{1}(t)$ is comprised of oscillation bursts separated by the excursions of $\tilde{a}_{1}(t)$ toward zero and is not a harmonic variation as one would infer from the SPOD result in Fig. 5(a). The mode shape shown in Fig. 5(c) now shows the spatial distribution of the amplitude associated with each of the oscillation bursts. Figure $5(d)$ shows the trajectory of the flow oscillations due to the first two WPOD modes that clearly dominate the flow dynamics at $\mathrm{St}=0.8$, in a phase space spanned by their respective instantaneous amplitudes computed using Eq. (7). The excursions toward the origin that this trajectory makes shows that the instantaneous amplitudes corresponding to both energetic WPOD modes approach zero simultaneously, confirming, therefore, that the flow oscillations are, in reality, intermittent and not harmonic and stationary as the SPOD result suggests.

The red contour in Fig. $5(d)$ shows the regions in the phase space visited by the trajectory $90 \%$ of the time within the 
measurement record. This is the contour of the joint probability density function of the modal amplitudes that encloses a region of $90 \%$ cumulative probability. We refer to this phase space region as the residence region of the trajectory. The change in the shape and position of the residence region helps identify broad changes in flow behavior as operating conditions change. An elongated residence region line in Fig. $5(d)$, extending toward the origin in phase space, also shows that the flow oscillations characterized by the two most energetic WPOD modes are intermittent. For a stationary coherent flow oscillation, it is easy to see that both the trajectory and residence region would compact and localized in the instantaneous amplitude phase space.

The above example shows how WPOD analysis can extract global features of nonstationary flow oscillations from flow field data that classical POD and SPOD analyses cannot resolve. Also, pointwise wavelet transforms such as the result in Fig. 4 are local, i.e., they give qualitative evidence of intermittent behavior at a point. In turbulent flows, this is broadly expected and does not necessarily imply global intermittent behavior, i.e., the whole flow oscillates intermittently. The WPOD analysis presented above provides precisely this type of insight as it constructs modes and their temporal evolution, directly from data that are representative of the global large-scale dynamics of the flow. We now apply the data analysis techniques discussed in this section to understand the impact of a centerbody on the unsteady dynamics of the flow in each of the cases shown in Table 1.

\section{Results and Discussion}

We first discuss the time averaged flow characteristics for each of the cases in Table 1 in order to understand the change in the position of the vortex breakdown bubble (VBB) relative to the centerbody and dump plane in each case. In the discussion that follows, the time average of a flow quantity $q$ is denoted by $\bar{q}$. SPOD and WPOD modes in the decomposition of $q$ will be denoted by $\hat{q}$ and $\tilde{q}$, respectively. All velocities have been normalized by the bulk flow velocity $U_{b}=8 \mathrm{~m} / \mathrm{s}$ at the exit of the inlet duct. The $x$ and $y$ directions will be referred to as streamwise and transverse directions, respectively (see Fig. 2).

Figures $6(a)-6(c)$ show the spatial variation of $\bar{U}_{x}$ for various values of centerbody diameter $D_{c}$, for the $S=0.67$ case (cases $1-3$ in Table 1). The black and magenta broken lines show the transverse extent of the inlet duct and the centerbody, respectively, in each case. The thick black contour corresponds to $\bar{U}_{x}=0$ in this and all other results presented in this section. Note that in all cases, the presence of a vortex breakdown bubble is clear. Figure $6(c)$ shows that removing the centerbody results in the emergence of a time averaged stagnation point in the flow, downstream of the dump plane. The effect of changing $D_{c}$ on $\bar{U}_{x}$ is to change the width of the VBB at its upstream end as the shape of the $\bar{U}_{x}=0$ contours in Figs. $6(a)-6(c)$ suggest, in addition to the appearance of an upstream stagnation point when the centerbody is removed. These results suggest that for $S=0.67$, the time averaged flow corresponds to a disjoint time averaged flow state with distinct CWRZ and VBB regions. Figures $6(d)-6(f)$ show $u_{\mathrm{RMS}}^{\prime}=\left[\left(u_{x, \mathrm{RMS}}^{\prime 2}+u_{y, \mathrm{RMS}}^{\prime 2}+u_{z, \mathrm{RMS}}^{\prime 2}\right) / 3\right]^{1 / 2}$, fields for each value of $D_{c}$. The impact of introducing the centerbody is seen to have a negligible impact on the spatial distribution of the turbulence intensity.

Figures 7(a)-7(c) show $\bar{U}_{x}$ fields for the $S=1.17$ cases (cases 4-6 in Table 1). Note that in all of these cases, the VBB is further upstream than in the $S=0.67$ cases (cases 1-3 in Table 1). Also, changing $D_{c}$ has a minimal impact on the VBB position and shape. Figure $7(c)$ suggests that the stagnation point due to the VBB is upstream of the dump plane. This means that for cases 4 and 5 (Figs. 7(a) and 7(b)), the VBB engulfs the upstream centerbody resulting in one continuous structure, resulting in a conjoined time average flow state. Figures $7(d)-7(f)$ show $u_{\text {RMS }}^{\prime}$ fields for each value of $D_{c}$ for $S=1.17$. Again, the impact of a centerbody on the $u_{\mathrm{RMS}}^{\prime}$ field is negligible in this case as well.

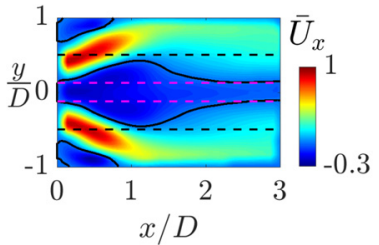

(a)

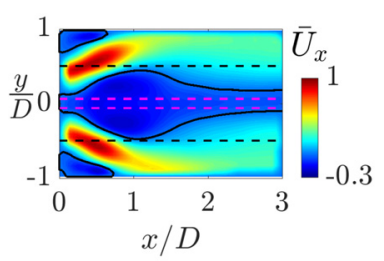

(b)

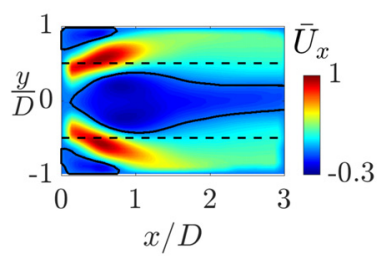

(c)

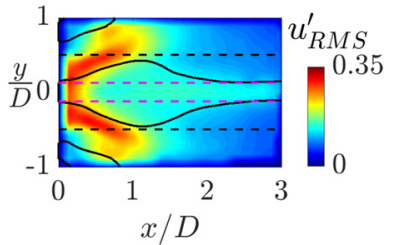

(d)

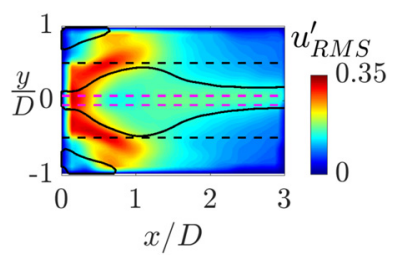

(e)

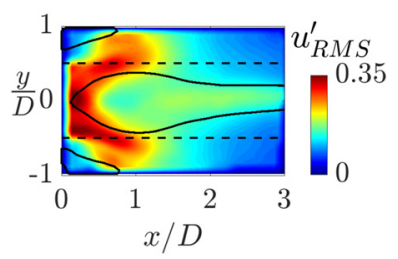

$(f)$
Fig. 6 Time averaged axial velocity $\left(\bar{U}_{x}\right)$ field for $S=0.67$ and (a) $D_{c}=9.5 \mathrm{~mm},(b) D_{c}=4.7 \mathrm{~mm}$, and $(c) D_{c}=0 \mathrm{~mm}$. RMS velocity $\left(u_{\text {RMS }}^{\prime}\right.$ ) field for $S=0.67$ and (d) $D_{c}=9.5 \mathrm{~mm},(e) D_{c}=4.7 \mathrm{~mm}$, and $(f) D_{c}=0 \mathrm{~mm}$. The fields have been normalized by $U_{b}$. The thick black contour on each figure shows $\bar{U}_{x}=0$. The black and magenta broken horizontal lines show the position of the inlet duct lips and edges of the centerbody, respectively, on either side of flow axis.

We next perform SPOD of the time series data in both cases to gain insight into the unsteady global dynamics of the flow in each of the above cases. These results have been obtained using $M=9$ ensembles comprised of $L=400$ snapshots with $50 \%$ overlap between successive snapshots. Figures $8(a)$ and $8(b)$ show the variation of the modal energy, $\hat{\sigma}_{0}^{2}$, with $\mathrm{St}=f D / U_{b}$ for different $D_{c}$ values for $S=0.67$ and 1.17 cases, respectively. The $S=0.67$ result (Fig. 8(a)) shows self-excited flow oscillations at $\mathrm{St} \sim 0.5$ for $D_{c}=0$ and $4.7 \mathrm{~mm}$ and a weaker flow oscillation that is $\sim 3$ times weaker at approximately the same value of $S t$ for $D_{c}=9.5 \mathrm{~mm}$. The $S=1.17$ case shows self-excited oscillations for the $D_{c}=0$ case at $\mathrm{St} \sim 0.8$ and weaker oscillations for both cases with centerbodies-see Fig. 8(b). We refer to the value of $S t$ for the most energetic SPOD mode for each combination of $S$ and $D_{c}$ shown in Table 1 as $\mathrm{St}_{\text {peak }}$ hereafter.

The real part of the $\hat{u}_{y}$ and $\hat{u}_{x}$ components of the most energetic SPOD modes at these values of $S t$ for the $D_{c}=0$ cases is shown in Fig. 9 for $S=0.67$ and in Fig. 10 for $S=1.17$. Note that there is a strong centerline $\hat{u}_{y}$ oscillation at the base of the VBB in both cases-see Figs. 9(a) and $10(a)$. Note also that the $\hat{u}_{y}$ oscillations on either side of the flow centerline are in phase while those corresponding to $\hat{u}_{x}$ are out of phase. This confirms the helical nature of the flow oscillation. The strong centerline $\hat{u}_{y}$ oscillation causes the base of the VBB to be deflected away from the geometric centerline. Combined with the presence of swirl, it is easy to see that this results in a strong precession of the VBB about the flow axis. The helical rollup of the shear layer between the VBB and the annular jet due to VBB precession can be seen from velocity oscillations along the shear layer in both Figs. $9(a)$ and $9(b)$ and $10(a)$ and $10(b)$. Therefore, all of these characteristics show that the self-excited oscillation is a PVC oscillation in the flow with a natural frequency $\mathrm{St}_{\text {peak }}$. Similar SPOD mode characteristics as 


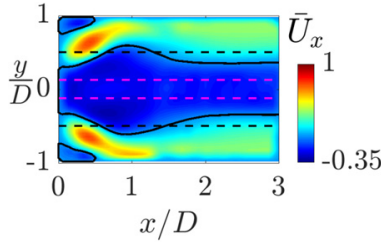

(a)

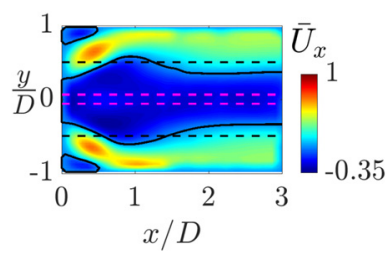

(b)

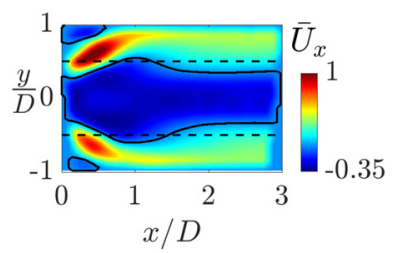

(c)

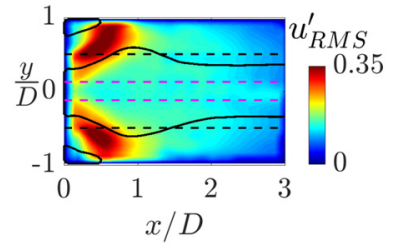

(d)

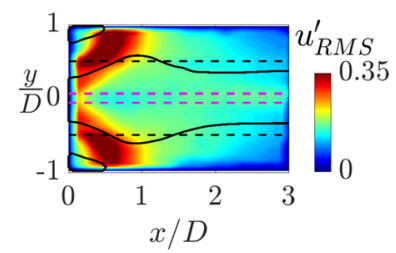

(e)

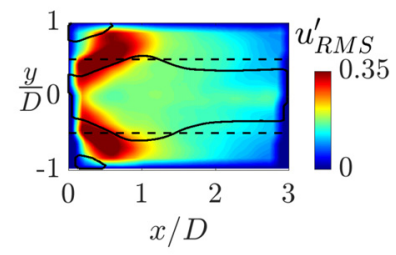

$(f)$
Fig. 7 Time averaged axial velocity $\left(\bar{U}_{x}\right)$ field for $S=1.17$ and (a) $D_{c}=9.5 \mathrm{~mm}$, (b) $D_{c}=4.7 \mathrm{~mm}$, and (c) $D_{c}=0 \mathrm{~mm}$. RMS velocity ( $u_{\mathrm{RMS}}$ ) field for $S=1.17$ and $(d) D_{c}=9.5 \mathrm{~mm},(e) D_{c}=4.7 \mathrm{~mm}$, and $(f) D_{c}=0 \mathrm{~mm}$. The fields have been normalized by $U_{b}$. The thick black contour on each figure shows $\bar{U}_{x}=0$. The black and magenta broken horizontal lines show the position of the inlet duct lips and edges of the centerbody, respectively, on either side of flow axis.

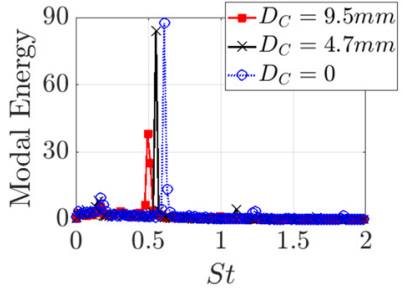

(a)

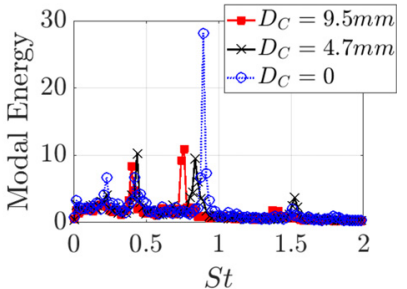

(b)
Fig. 8 Variation of the energy of the most energetic spectral POD mode with $S t=f D / U_{b}$ for $(a) S=0.67$ and $(b) S=1.17$ for each of the three values of $D_{c}$

shown in Figs. 9 and 10 are seen for cases with centerbodies as well at both values of $S$.

The increase in $\mathrm{St}_{\text {peak }}$ with $S$ is well known and has been reported in many prior studies [7,8,17]. Manoharan et al. [8] show using weakly nonlinear stability methods that the PVC is a stable limit cycle that emerges in a variable swirl jet when $S$ exceeds a critical value at which vortex breakdown occurs. For these values of $S$, a helical linearly marginally unstable mode with velocity oscillations concentrated at the centerline alone is present in the flow and results in the emergence of limit cycle hydrodynamic fluctuations. This shows that the fundamental instability mechanism driving the PVC is the precession of the VBB. The helical shear layer roll up, evident in Figs. $9(a), 9(b), 10(a)$, and $10(b)$, is a response to the unsteady rearrangement of the time averaged vorticity field, caused by VBB precession.

We now show that the low-amplitude oscillations in the cases with centerbodies shown in Fig. 8 are in reality intermittent, using

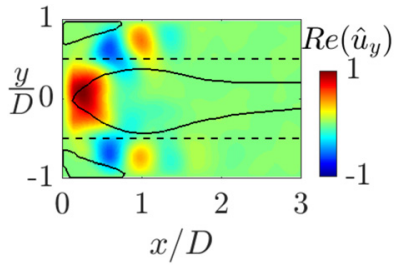

(a)

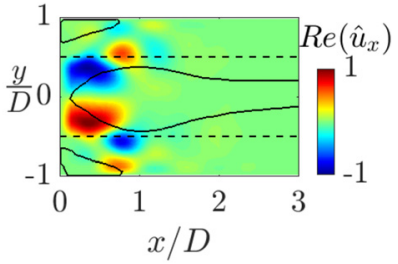

(b)
Fig. 9 Real parts of spectral POD modes at $\mathrm{St}_{\text {peak }}$ of (a) transverse, $\hat{u}_{y}$, and $(b)$ streamwise, $\hat{u}_{x}$, velocity components $\left(S=0.67, D_{c}=0.0 \mathrm{~mm}\right)$

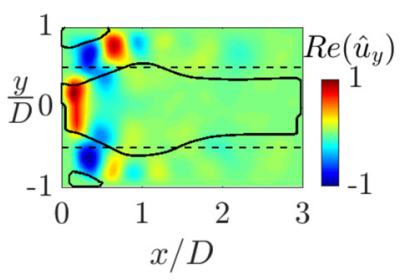

(a)

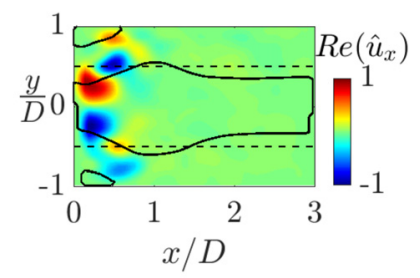

(b)
Fig. 10 Real parts of spectral POD modes at $\mathrm{St}_{\text {peak }}$ of (a) transverse, $\hat{u}_{y}$, and $(b)$ streamwise, $\hat{u}_{x}$, velocity components $\left(S=1.17, D_{c}=0.0 \mathrm{~mm}\right)$

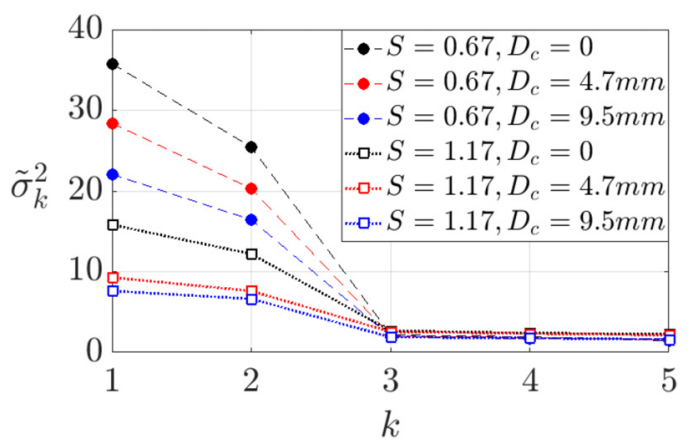

Fig. 11 Wavelet POD modal energy spectrum for all cases

WPOD analysis. Figure 11 shows the variation of the WPOD modal energies with mode number for $S=0.67$ (filled circles) and $S=1.17$ (empty squares). Note that in every case, the energy associated with the first two WPOD modes that recover PVC oscillations is greater than those of the remaining modes. The nominally lower energy in the case of $S=1.17, D_{c}=0$ when compared to $S=0.67$ is due to the upstream movement of the VBB with the increasing swirl. This movement results in the region of most energetic oscillations in the $S=1.17$ moving into the inlet duct and, therefore, out of the field of view of the present imaging system. The reduction in PVC oscillation energy with the introduction of centerbodies is also clear for both cases, showing that there is an overall stabilizing effect. However, the $S=0.67$ case shows a smooth reduction in the modal energy with increasing $D_{c}$, while the corresponding results for $S=1.17$ show similar modal energies. This suggests that the mechanism leading to intermittent oscillations in the two cases is qualitatively different as the size of the centerbody seems to have no impact on the oscillation intensity for the $S=1.17$ case.

Figures $12(a)$ and 12(b) show the most energetic WPOD mode of the transverse velocity field $\left(\tilde{u}_{y}\right)$ for $D_{c}=4.7 \mathrm{~mm}$ and $9.5 \mathrm{~mm}$, respectively, for $S=0.67$. Note that these modes represent the spatial amplitude distribution at $\mathrm{St}_{\text {peak }}$, corresponding to the intermittent burst oscillations. The close qualitative match between the 


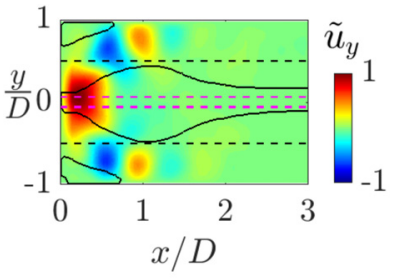

(a)

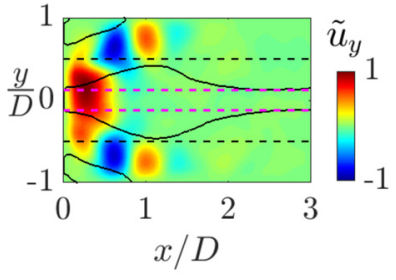

(b)
Fig. 12 Most energetic wavelet POD modes of the transverse velocity component $\left(\tilde{u}_{y}\right)$ for $S=0.67$ at $S t_{\text {peak }}$, (a) $D_{c}=4.7 \mathrm{~mm}$ and (b) $D_{c}=9.5 \mathrm{~mm}$. The black contours show $U_{x}=0$ for reference.

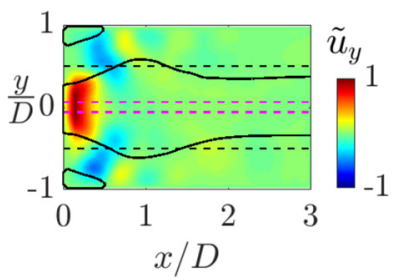

(a)

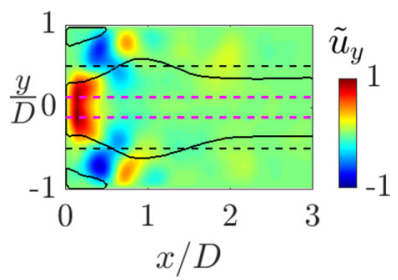

(b)
Fig. 13 Most energetic wavelet POD modes of the transverse velocity component $\left(\tilde{u}_{y}\right)$ for $S=1.17$ at $S t_{\text {peak }}$, (a) $D_{c}=4.7 \mathrm{~mm}$ and (b) $D_{c}=9.5 \mathrm{~mm}$. The black contours show $\tilde{U}_{x}=0$ for reference.

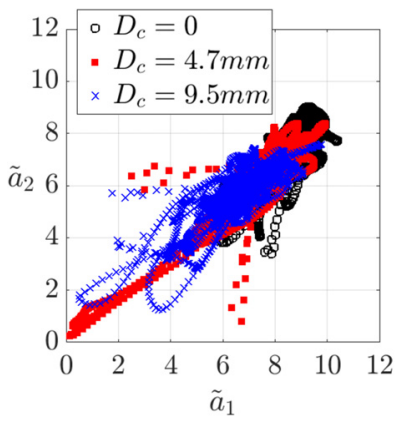

(a)

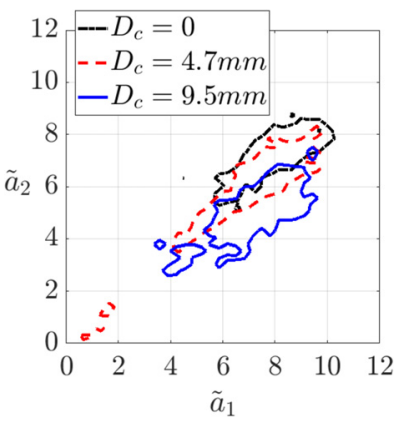

(b)
Fig. 14 Dynamics of modal oscillations in phase space for $S=0.67$ : (a) trajectory and $(b) 90 \%$ residence region

WPOD mode spatial amplitude distributions in Fig. 12 for $D_{c}>0$, with the SPOD mode from the corresponding $D_{c}=0$ case (Fig. 9(a)), shows that the intermittent oscillations correspond to helical PVC oscillations. Figures 13(a) and 13(b) show the most energetic WPOD modes for $D_{c}=4.7 \mathrm{~mm}$ and $9.5 \mathrm{~mm}$, respectively, for $S=1.17$. Again, the WPOD mode shapes shown in Figs. 13(a) and 13(b) and the SPOD mode shape from the corresponding $D_{c}=0$ shown in Fig. 10(a) compare well qualitatively. Therefore, for $S=1.17$ as well, the intermittent oscillations correspond to PVC oscillations. Although not shown, the comparison between SPOD and WPOD mode shapes for other velocity components shows the same quality of agreement for both $S=0.67$ and 1.17 .

Figures $14(a)$ and $14(b)$ show the phase trajectory of the flow oscillations in the instantaneous amplitude phase space, determined using Eq. (7) for the $S=0.67$ case, for each of the three $D_{c}$ values. The $90 \%$ residence region for $D_{c}=0$ in Fig. $14(b)$ shows that the trajectory of the flow oscillation stays mostly localized at large modal amplitudes. This shows that the PVC oscillations for this case are largely coherent, and therefore, the trajectory remains localized. The trajectories of the cases with centerbodies on the other hand show excursions toward the origin-see Fig. 14(a), showing that the intensity of the PVC oscillation intermittently reduces to very small values. Comparing Figs. 14(a) and 14(b) shows that the phase space regions associated with these excursions lie outside the residence region of their corresponding trajectories, further confirming that these excursions represent intermittent events. Figure 14(b) also shows a systematic shift of the residence regions toward the origin and lower instantaneous amplitudes with increasing $D_{c}$ for $S=0.67$.

Figures $15(a)$ and 15(b) show the trajectories and residence regions, respectively, for $S=1.17$ case for each of the three centerbody geometries. Note that even for this $S$, the cases with centerbodies show excursions toward the origin, confirming thereby the intermittent nature of flow oscillations in this case as well. However, unlike in the $S=0.67$ case, Fig. $15(b)$ shows that the residence regions for both cases with centerbodies are similar in shape and extent. Their similar position in phase space suggests a similar reduction in flow oscillation intensity relative to the $D_{c}=0$ case.

We now discuss the reasons for the observed instantaneous amplitude phase space trajectory characteristics. Mukherjee et al. [19] have shown for the disjoint case; merger of the CWRZ and $\mathrm{VBB}$ causes the linear hydrodynamic VBB precession mode to become marginally stable. However, intermittent VBB-CWRZ merger and separation can occur due to the presence of background turbulence. This can then lead to the growth of PVC oscillations. The amplitude attained by these oscillations then increases with the increase in the time interval between separation and merger events. Thus, the shift in the residence toward the origin suggests that this interval becomes smaller with increasing $D_{c}$-see Fig. 14(b). The nominal CWRZ length reduces when $D_{c}$ is reduced, which, in turn, makes CWRZ-VBB merger less likely, once separation has occurred, thereby, allowing more growth of the PVC oscillation amplitude as the result in Fig. 14(b) suggests. Therefore, the systematic shift of residence regions toward lower amplitudes (see Fig. 14(b)) and lower WPOD modal energies (see Fig. 11(a)) suggests that this is, indeed, the mechanism controlling intermittent PVC oscillations in the $S=0.67$ case. While it would be interesting to show evidence of these separation and merger events from instantaneous snapshots of the velocity field, it is not possible here due to the fact that the details of the flow near the dump plane are obscured by wall reflections making reliable visualization of the CWRZ difficult. One possible way to address this is to examine snapshots from LES simulations of this geometry. This is, however, beyond the scope of the study discussed in this paper.

In the conjoined $S=1.17$ case, the wavemaker of the linear hydrodynamic VBB precession mode is disturbed by the physical presence of the centerbody itself, rather than its wake, making it marginally stable. Farrell and Ioannou [34] show that for such flows, broadband forcing imposed on the time averaged mean

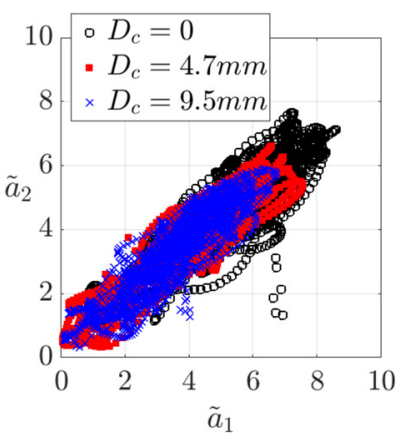

(a)

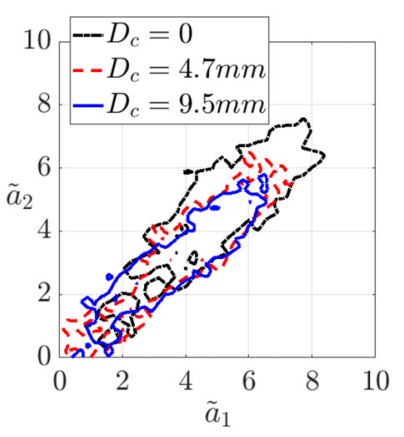

(b)
Fig. 15 Dynamics of modal oscillations in phase space for $S=1.17$ : (a) trajectory and $(b) 90 \%$ residence region 
flow state can result in intermittent oscillations at natural frequencies corresponding to stable hydrodynamic modes. As discussed earlier for the present experiments, the presence of the centerbody or its diameter $D_{c}$ has a negligible impact on the turbulence intensity distribution in the flow for $S=1.17$-see Figs. $7(d)-7(f)$. This suggests that the strength of the stochastic forcing imposed by turbulence is similar for both $D_{c}=4.7 \mathrm{~mm}$ and $D_{c}=9.5 \mathrm{~mm}$ at $S=1.17$. Therefore, it is reasonable to expect that the hydrodynamic response to forcing by turbulence would be similar for these two values of $D_{c}$. This is borne out by the similar modal energies of the WPOD modes, as well as, residence regions of similar position and shape for these two cases-see Figs. 11 and $15(b)$. These results, therefore, lend support to the conclusion that the stochastic hydrodynamic response of the marginally stable linear hydrodynamic VBB precession mode of the flow results in intermittent PVC oscillations for the conjoined $S=1.17$ cases.

Thus, in summary, the present results show two routes to intermittent PVC oscillations. For the disjoint $S=0.67$ case, the present results confirm the findings of the study of Mukherjee et al. [19], which showed that a merger between the VBB and CWRZ can result in the suppression of PVC oscillations. However, turbulent fluctuations can cause these two structures to intermittently separate and rejoin, resulting in PVC oscillation growth in the interval between these two events. In the conjoined $S=1.17$ case, stochastic forcing by turbulence of the time average flow with a stable linear hydrodynamic VBB precession mode can result in a hydrodynamic response driving intermittent PVC oscillations.

These intermittent PVC oscillations can then interact with the flame in various ways, potentially influencing combustor performance as discussed in the introduction. An interesting question for future study would be to understand the relationship between the spatial distribution of turbulence intensity and extent of stabilization of the linear hydrodynamic VBB precession mode on its receptivity to stochastic forcing by turbulence in the conjoined case.

\section{Conclusions}

This paper performs an experimental study on the impact of a centerbody on the dynamics of the PVC oscillation that occurs in gas turbine combustor swirl nozzles. These PVC oscillations are driven by the precession of the vortex breakdown bubble (VBB) due to a linearly unstable hydrodynamic mode that then results in the emergence of a stable limit cycle oscillation in the flow [8]. The presence of centerbodies is known to broadly result in the suppression of PVC oscillations. This is because they interfere with the wavemaker that is present at the upstream end of the VBB that drives PVC oscillations. Two time averaged flow states are possible in the presence of a centerbody, depending on the axial position of the vortex breakdown bubble (VBB) relative to the centerbody end face. The first is the disjoint state, wherein distinct CWRZ and VBB regions exist in the flow. The second state is the conjoined state where the VBB engulfs the centerbody, and no distinct CWRZ is observed.

In the disjoint case, Mukherjee et al. [19] show that merger between the CWRZ and VBB results in the stabilization of the linear VBB precession mode due to the disruption of the wavemaker. In the conjoined case, the physical presence of the centerbody suppresses the wavemaker and has the same effect on the stability of the linear mode [20]. The present nonreacting flow experimental study is performed by varying the centerbody diameter, $D_{c}$, for a fixed area averaged bulk flow velocity in a single nozzle swirl combustor. We study the flow state for two swirlers that generate flows with swirl numbers $S=0.67$ and $S=1.17$ in the combustor, corresponding to disjoint and conjoined time averaged flow states, respectively. Both cases show that intermittent PVC oscillations result when centerbodies are present. We show this using a relatively new modal decomposition technique, referred to as WPOD, that combines the frequency/time resolution property of wavelet transforms with the optimal mode extraction capability of the POD. While the focus in this paper is on analyzing data measured experimentally using sPIV, it is clear that the same technique can be used with no change to analyze other time resolved data, e.g., from time resolved LES as well.

Wavelet POD decompositions of data from cases with centerbodies for both swirl numbers show that the intermittent flow dynamics is characterized by a pair of modes that together represent PVC oscillations. The trajectories of flow evolution for these cases, reconstructed in phase space using the instantaneous amplitude of their respective WPOD modes, show excursions toward the origin. This shows that PVC oscillations in these cases are intermittent. For the $S=0.67$ case, the systematic shift of intermittent oscillation trajectories toward lower instantaneous amplitude regions in phase space with increasing $D_{c}$ suggests that intermittent oscillations are driven by separation and merger of the VBB and CWRZ. This results in the intermittent appearance of PVC oscillations in the time interval between these events. In the $S=1.17$ case, the similar shape and position of the phase space regions occupied by the trajectories suggests that PVC oscillations are driven by the response of the time averaged flow due to the VBB precession mode because of stochastic forcing by turbulence.

These results show that in practical swirl nozzles, passive nozzle geometry changes may not entirely suppress PVC oscillations. Intermittent PVC oscillations can still cause flame shape transitions and influence fuel-air mixing in a way that promotes thermoacoustic instabilities. In the disjoint case, present results suggest that complete suppression appears to be achievable by simply increasing the CWRZ length, i.e., by increasing the centerbody diameter. In the conjoined case, however, the path to achieving complete PVC suppression is not entirely clear. An interesting problem for future study is the relationship between the extent of stabilization of the linear VBB precession mode and the characteristics of the stochastic intermittent PVC response oscillation that may be excited. Insight of this kind can help drive nozzle design choices for next generation gas turbine combustors.

\section{References}

[1] Lefebvre, A. H., and Ballal, D. R., 2010, Gas Turbine Combustion: Alternative Fuels and Emissions, CRC Press, Boca Raton, FL.

[2] Benjamin, T. B., 1967, "Some Developments in the Theory of Vortex Breakdown,” J. Fluid Mech., 28(1), pp. 65-84.

[3] Sarpkaya, T., 1971, "On Stationary and Travelling Vortex Breakdowns," J. Fluid Mech., 45(3), pp. 545-559.

[4] Leibovich, S., 1978, "The Structure of Vortex Breakdown," Annu. Rev. Fluid Mech., 10(1), pp. 221-246.

[5] Liang, H., and Maxworthy, T., 2005, "An Experimental Investigation of Swirling Jets," J. Fluid Mech., 525, pp. 115-159.

[6] Escudier, M., and Keller, J., 1985, "Recirculation in Swirling Flow-a Manifestation of Vortex Breakdown," AIAA J., 23(1), pp. 111-116.

[7] Oberleithner, K., Sieber, M., Nayeri, C. N., Paschereit, C. O., Petz, C., Hege, H.-C., Noack, B. R., and Wygnanski, I., 2011, "Three-Dimensional Coherent Structures in a Swirling Jet Undergoing Vortex Breakdown: Stability Analysis and Empirical Mode Construction," J. Fluid Mech., 679, pp. 383-414.

[8] Manoharan, K., Frederick, M., Clees, S., O'Connor, J., and Hemchandra, S. 2020, "A Weakly Nonlinear Analysis of the Precessing Vortex Core Oscillation in a Variable Swirl Turbulent Round Jet,” J. Fluid Mech., 884, p. A29.

[9] Syred, N. 2006, "A Review of Oscillation Mechanisms and the Role of the Precessing Vortex Core (PVC) in Swirl Combustion Systems," Prog. Energy Combust. Sci., 32(2), pp. 93-161.

[10] Taamallah, S., Dagan, Y., Chakroun, N., Shanbhogue, S., Vogiatzaki, K., and Ghoniem, A. F., 2019, "Helical Vortex Core Dynamics and Flame Interaction in Turbulent Premixed Swirl Combustion: A Combined Experimental and Large Eddy Simulation Investigation," Phys. Fluids, 31(2), p. 025108.

[11] Shanbhogue, S., Sanusi, Y., Taamallah, S., Habib, M., Mokheimer, E., and Ghoniem, A., 2016, "Flame Macrostructures, Combustion Instability and Extinction Strain Scaling in Swirl-Stabilized Premixed CH4/H2 Combustion," Combust. Flame, 163, pp. 494-507.

[12] Taamallah, S., Shanbhogue, S. J., and Ghoniem, A. F., 2016, "Turbulent Flame Stabilization Modes in Premixed Swirl Combustion: Physical Mechanism and Karlovitz Number-Based Criterion," Combust. Flame, 166, pp. 19-33.

[13] Oberleithner, K., Stöhr, M., Im, S. H., Arndt, C. M., and Steinberg, A. M., 2015, "Formation and Flame-Induced Suppression of the Precessing Vortex Core in a Swirl Combustor: Experiments and Linear Stability Analysis," Combust. Flame, 162(8), pp. 3100-3114.

[14] Moeck, J. P., Bourgouin, J.-F., Durox, D., Schuller, T., and Candel, S., 2012 "Nonlinear Interaction Between a Precessing Vortex Core and Acoustic Oscillations in a Turbulent Swirling Flame," Combust. Flame, 159(8), pp. 2650-2668. 
[15] LaBry, Z. A., Shanbhogue, S. J., Speth, R. L., and Ghoniem, A. F., 2011, "Flow Structures in a Lean-Premixed Swirl-Stabilized Combustor With Microjet Air Injection," Proc. Combust. Inst., 33(1), pp. 1575-1581.

[16] Ghoniem, A. F., Labry, Z. A., Shanbhogue, S. J., and Speth, R. L., 2014 "Swirl-Counter-Swirl Microjets for Thermoacoustic Instability Suppression," U.S. Patent No. 8,708,696 B2

[17] Anacleto, P., Fernandes, E., Heitor, M., and Shtork, S., 2003, "Swirl Flow Structure and Flame Characteristics in a Model Lean Premixed Combustor," Combust. Sci. Technol., 175(8), pp. 1369-1388.

[18] Lückoff, F., Sieber, M., Paschereit, C. O., and Oberleithner, K., 2020, "Impact of the Precessing Vortex Core on NOx Emissions in Premixed Swirl-Stabilized Flames: An Experimental Study," ASME J. Eng. Gas Turbines Power, 142(11), p. 111010.

[19] Mukherjee, A., Muthichur, N., More, C., Gupta, S., and Hemchandra, S., 2021 "The Role of the Centerbody Wake on the Precessing Vortex Core Dynamics of a Swirl Nozzle," ASME J. Eng. Gas Turbines Power, 143(5), p. 051019.

[20] Kaiser, T. L., Oberleithner, K., Selle, L., and Poinsot, T., 2019, "Examining the Effect of Geometry Changes in Industrial Fuel Injection Systems on Hydrodynamic Structures With Biglobal Linear Stability Analysis," ASME J. Eng. Gas Turbines Power, 142(1), p. 011024.

[21] Tammisola, O., and Juniper, M. P., 2016, "Coherent Structures in a Swirl Injector at $\mathrm{Re}=4800$ by Nonlinear Simulations and Linear Global Modes," J. Fluid Mech., 792, pp. 620-657.

[22] Karmarkar, A., Tyagi, A., Hemchandra, S., and O'Connor, J., 2021, "Impact of Turbulence on the Coherent Flame Dynamics in a Bluff-Body Stabilized Flame," Proceedings of the Combustion Institute, 38(2), pp. 3067-3075.
[23] Yin, Z., and Stöhr, M., 2020, "Time-Frequency Localisation of Intermitten Dynamics in a Bistable Turbulent Swirl Flame," J. Fluid Mech., 882, p. A30.

[24] Mendez, M. A., Balabane, M., and Buchlin, J.-M., 2019, "Multi-Scale Proper Orthogonal Decomposition of Complex Fluid Flows," J. Fluid Mech., 870, pp. 988-1036.

[25] Beer, J., and Chigier, N., 1972, Combustion Aerodynamics, Applied Science Publishers, London, UK.

[26] Chatterjee, A., 2000, "An Introduction to the Proper Orthogonal Decomposition," Curr. Sci., 78(7), pp. 808-817.

[27] Berkooz, G., Holmes, P., and Lumley, J. L., 1993, "The Proper Orthogonal Decomposition in the Analysis of Turbulent Flows," Annu. Rev. Fluid Mech., 25(1), pp. 539-575.

[28] Weiss, J., 2019, "A Tutorial on the Proper Orthogonal Decomposition," AIAA Paper No. 2019-3333.

[29] Sirovich, L., 1987, "Turbulence and the Dynamics of Coherent Structures. I. Coherent Structures," Q. Appl. Math., 45(3), pp. 561-571.

[30] Towne, A., Schmidt, O. T., and Colonius, T, 2018 "Spectral Proper Orthogonal Decomposition and Its Relationship to Dynamic Mode Decomposition and Resolvent Analysis," J. Fluid Mech., 847, pp. 821-867.

[31] Sieber, M., Paschereit, C. O., and Oberleithner, K., 2016, "Spectral Proper Orthogonal Decomposition," J. Fluid Mech., 792, pp. 798-828.

[32] Grenander, U., and Szegö, G., 1958, Toeplitz Forms and Their Applications, University of California Press, Berkeley, CA.

[33] Percival, D. B., and Walden, A. T., 2000, Wavelet Methods for Time Series Analysis, Vol. 4, Cambridge University Press, Cambridge, UK.

[34] Farrell, B. F., and Ioannou, P. J., 1993, "Stochastic Forcing of the Linearized Navier-Stokes Equations," Phys. Fluids A: Fluid Dyn., 5(11), pp. 2600-2609. 